\title{
Le silence en maux dans l'œuvre théâtrale de Samuel Beckett
}

Le silence jalonne l'œuvre théâtrale de Beckett d'En attendant Godot à Acte sans paroles. Toujours, il y a cette même exigence de l'extinction progressive du verbe.

Les temps et autres pauses, qui émaillent le texte comme le ferait une ponctuation, matérialisent en premier lieu l'angoisse des personnages confrontés à la vacuité de leur existence. Ce chaos linguistique préfigurerait alors la fin. Les personnages éprouvent littéralement le silence comme le surgissement du néant, une sorte de passerelle vers la finitude qui contient donc toute l'angoisse existentielle. Ce silence est aussi celui qui s'installe au milieu des couples beckettiens pour traduire l'aporie du langage.

Cette impossibilité du dire est alors souvent, chez Beckett, remplacée par le faire. Celui-ci s'exprime dans une gestualité ostentatoire venant aussi bien donner une matérialité à ce silence que tenter désespérément de le combler. Les personnages du théâtre de Beckett agissent et donnent au silence une consistance, l'incarnent comme un personnage à part entière.

Pourtant, le silence peut aussi être perçu comme salutaire : il crée par la suspension du discours un effet d'attente. Espoir de l'autre, quête de l'échange et donc d'une forme de réciprocité. Présent pour témoigner de la vacuité du langage, de sa facticité, le silence est aussi le lieu du possible. Rien n'a encore été dit donc tout est à venir.

\section{Le silence comme surgissement du néant}

\section{De l'angoisse existentielle muette...}

Le silence apparait tout d'abord dans le théâtre de Beckett comme un marqueur de la finitude. Présent plus d'une centaine de fois dans les pièces principales, il agit comme une balise syntaxique au cœur du texte dit. Pour la plupart des figures becket-

Marjorie Colin - agrégée de lettres modernes et doctorante à l'Université Sorbonne Nouvelle - Paris 3. Adresse pour correspondance : marjoriecolin@yahoo.fr 
tiennes, ces silences renvoient à l'angoisse existentielle du personnage condamné à mourir. Parler devient alors, pour ces personnages en décrépitude, un moyen de résister, de ne pas mourir. De fait, le silence est donc dans un premier temps associé à la fin. Se taire, c'est mourir. Il s'agit alors de déverser des flots de paroles pour lutter contre cette angoisse du vide. Le silence s'inscrit donc dans cette logique de la finitude, car il renvoie le personnage au manque, à l'absence et à l'oubli. Cela est particulièrement sensible dans En attendant Godot où la nécessité de la parole est impérative. "Vladimir. - (Un temps.) Voyons, Gogo, il faut me renvoyer la balle de temps en temps. » (Beckett, 1952 : 15); et plus loin « Vladimir. - Veux-tu que je m'en aille? (Un temps). Gogo! (Un temps. Vladimir le regarde avec attention.) On t’a battu ? (Un temps). Gogo ! (Beckett, 1952 : 81). Le silence est un moyen pour Vladimir de chercher à capter l'attention de son interlocuteur, inquiet d'un silence définitif. C'est également le cas dans Oh les beaux jours, lorsque Winnie s'adresse à Willie :

Même quand tu seras parti, Willie. (Elle se tourne un peu vers lui.) Tu pars, Willie, n'estce pas? (Un temps. Se tournant un peu plus vers lui, plus fort.) Tu vas bientôt partir, Willie, n'est-ce pas? (Un temps. Plus fort.) Willie! (Un temps.) (Beckett, $1963: 34$ ).

Les marqueurs de suspension du discours que l'on retrouve par les expressions Un temps, Pause ou Silence illustrent une rupture dans la continuité discursive. Ici, l'interruption de la parole est un moyen pour le personnage d'inviter son interlocuteur à poursuivre, à rebondir sur la parole donnée, et plus qu'une invitation, il s'agit d'une exigence. Les questions successives sont une main tendue dans un espace vide. Mais c'est une main tremblante, effrayée par la possibilité du silence de l'Autre. Cette nécessité de l'échange apparaît comme un remède à la solitude et à l'angoisse métaphysique de la mort, même lorsque le personnage est seul en scène, comme l'est Krapp dans La dernière bande. L'auto-questionnement n'est qu'un moyen de montrer que parler est exister, un outil de lutte contre l'enfouissement du verbe : «Effie... (Pause.) Aurais pu être heureux avec elle là-haut sur la Baltique, et les pins, et les dunes. (Pause.) Non? (Pause.) Et elle? (Pause.)» (Beckett, 1959 : 29). C'est en quelque sorte la fonction aussi de la parenthèse (Un temps.) dans Oh les beaux jours : les trous dans le discours de Winnie marquent de manière obsessionnelle la nécessité du souvenir. C'est l'effort de la mémoire qui est tout entier contenu dans ces marqueurs du silence : on perçoit alors toute la difficulté du personnage pour faire surgir le passé dans son propre discours. Les silences véhiculent ici l'angoisse de Winnie pour reconstruire ses souvenirs. La mémoire hésitante implique une forme d’oubli, de marqueur macabre. Il faut parler pour essayer de se souvenir comme il faut se souvenir pour pouvoir parler. L'obsession de la parole est récurrente dans le théâtre beckettien, même si cette parole s'amenuise de pièce en pièce. Ainsi les deux " long silence " qui précèdent les répliques impératives de Vladimir « Dis quelque chose ! " puis "Vladimir (angoissé). - Dis n'importe quoi!» (Beckett, 1952 : 88) illustrent cette urgence d'une parole résistante face à l'inexorable fatalité de l'homme condamné à mourir. De fait, 
le silence apparait comme le surgissement du néant et vient matérialiser ce vide que les personnages vont chercher, par la parole, à combler.

\section{2 ... à l'aporie du langage}

Du marquage symbolique de la finitude, ces silences vont ensuite s'étendre aux fonctions du langage pour traduire l'échec de la communication. Seule la fonction phatique paraît être épargnée. La parole semble alors se rétracter sur le personnage qui parle, lui revenir, comme pour témoigner de l'impossibilité de la communication. Deux expressions de cette aporie sont manifestes dans le théâtre de Beckett : l'échec de la parole de soi à soi, l'échec de la communication à deux.

Dans le rapport que le personnage entretient avec son propre discours, on constate que les silences témoignent d'une suspension, d'une parole qui syntaxiquement déraille. La continuité discursive est impossible tant les silences viennent trouer la linéarité de la parole. Les pauses et autres temps éclatent le discours, le fragmentent, marquant, par moment, la folie d'un personnage empêtré dans un verbe délirant, comme celui du Dr Piouk d'Eleutheria:

J'exposais hier, devant le regretté monsieur Krap, homme remarquable d'ailleurs, à sa manière, ma façon d'envisager le problème de l'existence humaine, car c'est un problème, à mon avis, malgré les efforts qu’on fait aujourd'hui pour démontrer le contraire. (Pause.) Je dirai même que je n'en vois pas d'autre. (Pause.) N'étant pas une fourmi, par exemple, ou une baleine. (Pause.) (Beckett, 1995 : 106).

Les silences viennent baliser les différents temps d'une parole qui achoppe autant que l'esprit semble s'égarer. Dans La dernière bande, Krapp, seul personnage de la pièce, interrompt son récit à maintes reprises : "(Pause.) Et ainsi de suite. (Pause.) Sois de nouveau, sois de nouveau. (Pause.) Toute cette vieille misère. (Pause.) Une fois ne t'a pas suffit. (Pause.) Coule-toi sur elle.» (Beckett, 1959: 31). On perçoit ici toute la difficulté d'assurer une cohérence logique à un discours grêlé de pauses ou d'aposiopèses. Ces silences récurrents illustrent donc une parole en souffrance, contaminée par l'essoufflement du personnage. La logique discursive, annulée par la surcharge des silences, renvoie, en effet, à la décrépitude psychique du personnage. Ce déraillement de la parole, interrompue sans cesse par les pauses, évoque une fatigue manifeste : «Boîte - (Pause.) trois, bobine - (Pause.) cinq (Pause.)» (Beckett, 1959 : 31). Krapp semble en proie à des difficultés d'ordre physique et psychique qui se propagent au verbe. Tout devient laborieux, du geste à la parole, du corps à l'esprit. C’est également le cas dans Fin de partie:

Pozzo (avec colère). - Ne me coupez pas la parole! (Un temps. Plus calme.) Si nous parlons tous en même temps, nous ne nous en sortirons jamais. (Un temps.) Qu'est-ce que je disais? (Un temps. Plus fort.) Qu'est-ce que je disais? (Beckett, 1957 : 16). 
Selon Luc Vigier, « [m]odalité suspensive, la parenthèse (Un temps.) est aussi coupure, faille, déchirure et menace - par ses effets de perforations et de découpe - la tenue même du discours ou de la pensée suivie » $(2010: 66)$.

Mais cette aporie de la communication est aussi manifeste dans les échanges à deux. Ainsi, Estragon et Vladimir ont de véritables dialogues de sourds, marqués par les silences qui viennent matérialiser l'incompréhension d'un des personnages : "Estragon : Je ne comprends rien... (Un temps.) ». Plus loin dans la pièce, Vladimir affirme l'inutilité de la parole « Ne perdons pas notre temps avec de vains discours. » (Beckett, 1952 : 111) avant, prétérition, d’entamer « avec véhémence » une tirade qui s'avèrera ne produire absolument aucun effet sur Estragon : "Qu'en dis-tu ? (Estragon n'en dit rien.)» (Beckett, 1952 : 112). L'incompréhension est similaire dans Eleutheria :

Mlle Skunk. - Il me fait la cour. (Silence.) Tu sais ce qu'il m'a dit? (Silence. Mlle Skunk piaffe.) Mais réponds pour une fois!

Victor. - Je ne comprends pas. (Beckett, 1995 : 95)

D’autre part, les silences viennent là aussi interrompre la logique de l'échange et la linéarité du propos :

Hamm. - Alors c'est une journée comme les autres.

Clov. - Tant qu'elle dure. (Un temps.) Toute la vie les mêmes inepties. (Un temps.)

Hamm. - Moi je ne peux pas te quitter. (Beckett, 1957 : 64)

Le silence marque donc une rupture dans la linéarité dramatique, un refus de la logique discursive, l'essentiel n'étant pas de rendre compte d'une cohérence dramatique mais de parler pour meubler. On assiste ainsi, par l'intermédiaire de ces silences qui agissent comme des marqueurs syntaxiques, à un bondissement du verbe qui enjambe littéralement ces silences pour aller d'un sujet à un autre. On perçoit ici la fonction d'un silence qui vient briser l'ensemble logique pour donner l'image d'une parole éclatée, fragmentée, d'une véritable impasse dans la communication.

\section{Le corps, relais parlant d'un verbe muet}

\subsection{Double mise en échec de la parole et du silence}

On peut considérer qu'il y a, dans le théâtre de Beckett, une double mise en échec, à la fois du silence et de la parole. Si l'on peut penser que le discours est troué de silences, sans doute est-il aussi juste de dire que le silence est troué de paroles. Faut-il comprendre par là que le langage vient empêcher le silence d'être total, devenant alors un obstacle dans ce désir d'extinction du verbe ? Dans l'ensemble des pièces, il est question plusieurs fois d'une parole que le silence vient annuler ou, au contraire, 
d'un silence que la parole vient remplir. L'exigence du silence est impossible. La parole est incontinente. Et lorsque le texte didascalique annonce un temps ou une pause, la mention même d'un discours, qui renvoie à l'impératif de se taire, vient contredire d'emblée ce jeu du silence :

Il en va de même du rire. (Il rit). Ne disons pas de mal de notre époque, elle n'est pas plus malheureuse que les précédentes. (Silence.) N’en disons pas de bien non plus. (Silence.) N’en parlons pas. (Silence.) (Beckett, $1952: 45$ ).

On perçoit ici toute la contradiction entre parole et silence. Les verbes dire et parler témoignent, à la forme négative, de ce refus du verbe, renforcé par la mention didascalique du silence. Pour autant, ces pauses sont suivies immédiatement d'un nouvel acte de parole qui vient anéantir l'exigence formulée par le personnage. «Beckett ne cesse de se confronter à cette aporie : comment dire le silence au moyen de mots, alors même que celui-ci pour exister exige la disparition des mots? " (Siboni, 2010 : 18). Dans les exemples cités, le marqueur didascalique silence vient annoncer la parole qui exige le silence alors même qu'elle le nie au moment où la bouche parle. Dire je me tais n'est pas se taire. Il en résulte donc une impossibilité de ne pas parler. De fait, cet espace intermédiaire, entre parole et silence, cette zone blanche, pourrait symboliser l'incertitude humaine, l'incapacité à choisir entre le flot verbal continu et l'interruption définitive. Véritable clé de voûte du théâtre beckettien, cette croisée, à l'instar des croisées d'ogives dans les cathédrales, soutiendrait toute l'armature dramatique des pièces : ni parler ni se taire. Ni vivre ni mourir. La parole devient acte de résistance quand le silence est là pour rappeler le surgissement de notre finitude. Cette impossibilité d'un silence total est particulièrement sensible dans Esquisse radiophonique:

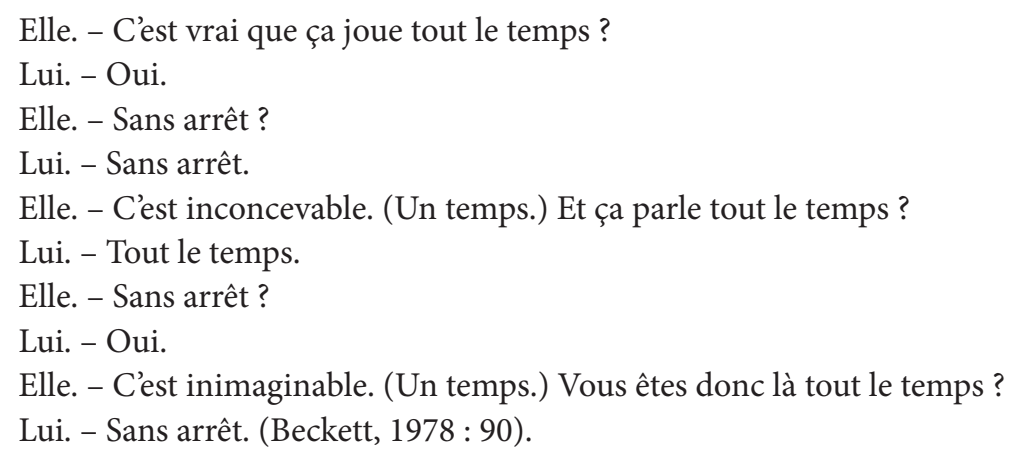

Ce balancement permanent entre parole et silence marque l'oscillation humaine et l'inconstance métaphysique des personnages : "Allons-y. (Ils ne bougent pas.) " (Beckett, 1952 : 134) en est sans doute l'exemple le plus saillant. Placé à la toute fin de Godot, cela peut symboliser une clé de lecture de l'ensemble du texte. Le corps 
vient ici contredire le verbe, voire le remplacer, comme acte de langage à part entière. Comme l'écrit Luc Vigier, « [...] la didascalie (Un temps.) coupant la parole, placée comme incrustation à effet de suspension, crée secrètement un discours autonome d'où la tentation du jeu n'est pas absente [...]» (2010:63). De fait, c'est bien le geste qui va se substituer au verbe pour parler à son tour, pour parler à sa place. Lorsque les personnages ne disent plus, ils font.

\subsection{Le silence : la parole du corps}

En effet, nous pouvons considérer que le silence s'exprime notamment par la profusion des didascalies gestuelles qui interrompent le dialogue théâtral. La gestualité peut donc apparaitre comme parole du corps. Puisque le silence s'impose sur scène, il faut le combler par l'action. Elle seule est capable de donner de l'espoir aux personnages. Agir, c'est éviter de mourir. Le silence n'est donc pas circonscrit aux seules mentions des pauses ou des temps. Le corps vient ainsi emplir l'espace verbal laissé béant. Agir permet donc en quelque sorte de remédier à l'extinction de la voix. À la parole agonisante, le corps surgit en remplaçant, de la même manière qu'au corps décrépit répond la parole. Les personnages de Beckett se situent toujours au carrefour des deux voies : lorsque le verbe ne peut plus, c'est bien le corps qui se charge de témoigner d'une forme de résistance contre la finitude, c'est bien le corps qui véritablement parle. L'exigence du faire traduit cette ténacité. Ainsi, dans Oh les beaux jours, les didascalies nombreuses concernant les gestes de Winnie viennent supplanter la parole difficile et l'échange impossible avec Willie :

(Elle se tourne un peu vers lui.) Tu sais le rêve que je fais quelques fois? (Un temps.) Le rêve que je fais quelques fois, Willie ? (Un temps.) Que tu viendras vivre de ce côté que je puisse te voir. (Un temps. Elle revient de face.) J’en serais transformée. (Un temps.) Méconnaissable. (Elle se tourne un peu vers lui.) Ou seulement de temps en temps, de ce côté seulement de temps en temps, que je me repaisse de toi. (Un temps. Elle revient de face.) Mais tu ne peux pas, je sais. (Elle baisse la tête.) Je sais. (Elle lève la tête.) Enfin - (Elle regarde la brosse) - plus pour longtemps, Winnie - (elle regarde la brosse) - ça va sonner. (Beckett, 1963 : 55).

On perçoit ici toute la puissance verbale de la gestualité qui vient dialoguer avec le personnage, comme pour remplacer le vide laissé par la parole de l'autre. Cette surcharge du geste permet de combattre l'angoisse du vide. Puisque le dialogue est impossible, puisque la parole se fait agonisante, le corps apparait donc en béquille d'un langage verbal défaillant. C'est évidemment encore plus manifeste dans Actes sans paroles, mimodrames qui marquent l'absence totale de langage verbal, exhibant littéralement ce silence par les gestes, nombreux, itératifs, compulsifs. Le corps devient ainsi le levier d'un langage particulier qui témoigne de l'impossibilité du verbe. Beckett poursuit ici un but précis : annuler totalement la parole, montrer l'effondrement progressif du langage verbal au profit, comme l’écrit Artaud, du « langage 
des signes, des gestes et des mimiques " $(1964: 37)$. « Le mot est une fuite. Le mot empêche le silence de parler ", écrit Ionesco dans Journal en miettes (1967 : 121). Sans doute faut-il en conclure que chez Beckett, le geste permet finalement au silence de dire quelque chose.

\section{Les promesses du silence}

\subsection{Déconstruire la facticité du langage}

Le silence apparaît in fine comme un élément indispensable pour déconstruire les clichés, les lieux communs et autres truismes langagiers. C'est ce que souligne Emmanuel Jacquart à propos de la pièce Oh les beaux jours : « Le silence systématisé voilant à dessein le message est un aspect de la fragmentation qui élimine certains détails, abolit les rapports entre les éléments traditionnels du discours et ne laisse subsister que l'essentiel » (1998: 220). Beckett, à travers l'usage récurrent des pauses et autres temps, s'attache à mettre au jour la facticité d'un langage qui n'exprime rien, raison pour laquelle il lui préfère le silence. Cela est assez manifeste dans Godot, notamment dans la tirade de Lucky qui présente de façon exceptionnelle une réplique extrêmement longue sans aucune pause, ce qui chez Beckett fait véritablement exception. Sans doute faut-il y voir justement un argument majeur pour témoigner de la facticité du langage, éminemment plus saillante ici que dans les répliques qui mentionnent régulièrement les silences. Autrement dit : moins il y a de silence, plus le discours est inintelligible et creux. La jargonaphasie témoigne, dans cette réplique, d'un assemblage totalement décousu et délirant :

[...] il est établi tabli tabli ce qui suit qui suit qui suit assavoir mais n'anticipons pas on ne sait pourquoi à la suite des travaux de Poinçon et Wattmann il apparait aussi clairement si clairement [...] que l'homme enfin malgré les progrès de l'alimentation et et de l'élimination des déchets est en train de maigrir [...]. (Beckett, $1952: 60$ ).

Cet amas de phrases disparates marque une forme de démence dans laquelle se déploie une puissante charge comique. Assumée sérieusement par le personnage, sorte de parodie de discours journalistico-socio-philosophique, cette tirade n'est en réalité qu'une succession de phrases assemblées en collage pour renforcer toute l'absurdité d'un langage qui n'exprime finalement rien. Pozzo apparaît également comme le personnage dont la vacuité du discours est sans doute la plus manifeste et s'exprime cette fois au milieu des silences :

Les larmes du monde sont immuables. Pour chacun qui se met à pleurer, quelque part un autre s'arrête. (...) Ne disons pas de mal de notre époque, elle n'est pas plus malheureuse que les précédentes. (Silence.) N’en disons pas de bien non plus. (Silence.) N’en parlons pas. (Silence.) Il est vrai que la population a augmenté. (Beckett, $1952: 45$ ). 
Cette saillie finale témoigne d'une association d'idées convenues que le dramaturge tente de brutaliser par la mention répétée des silences qui agissent comme des avertisseurs. De fait, la vacuité du langage est mise en évidence par ces interruptions dans le discours comme pour en souligner la portée finalement dérisoire.

Les mots signifient-ils quelque chose, du moins peuvent-ils dire quelque chose, semble demander Beckett ? C'est à peu près ce qui ressort de l'échange entre Hamm et Clov :

Hamm. - (Un temps. Avec angoisse.) Clov!

Clov. - Oui.

Hamm. - Qu'est-ce qui se passe?

Clov. - Quelque chose suit son cours.

Un temps.

Hamm. - Clov !

Clov (agacé). - Qu'est-ce que c'est ?

Hamm. - On n'est pas en train de... de... signifier quelque chose?

Clov. - Signifier ? Nous, signifier ! (Rire bref.) Ah elle est bonne! (Beckett, 1957 : 49).

Ainsi le silence permet ici, de manière salvatrice, d'évacuer le vide du langage tout en en dénonçant les stéréotypes. Il jette alors un discrédit total sur la parole, ce que souligne Lydie Parisse lorsqu'elle rapporte ces propos :

Étant donné que nous ne pouvons éliminer le langage d'un seul coup, nous devons au moins ne rien négliger de ce qui peut contribuer à son discrédit. Y forer des trous, l'un après l'autre, jusqu'au moment où ce qui est tapi derrière, que ce soit quelque chose ou rien du tout, se mette à suinter de travers (Parisse, $2008: 21$ ).

Le silence apparaît donc comme un instrument puissant pour déconstruire le langage, le remettre en cause et s'interroger sur la finalité de ses fonctions et de son pouvoir, ce que souligne la réplique de Clov dans Fin de partie :

Clov. - (Un temps.) Puis un jour, soudain, ça finit, ça change, je ne comprends pas, ça meurt, ou c'est moi, je ne comprends pas, ça non plus. Je le demande aux mots qui restent - sommeil, réveil, soir, matin. Ils ne savent rien dire. (Un temps.) (Beckett, 1957 : 108-109).

Le silence tient donc sa promesse, et si l’enjeu est bien d'anéantir les clichés langagiers et la vacuité de la parole, il ne faut pas négliger les répliques souvent trouées qui peuvent aussi apparaître comme des manifestations d'un langage poétique puissant. Si Beckett pratique la démolition de la fonction référentielle, la fonction poétique semble toutefois, quant à elle, résister. C'est une des promesses aussi de ce silence beckettien, qui offre à la parole une forme de résonance, par la mise en relief de certains mots : 


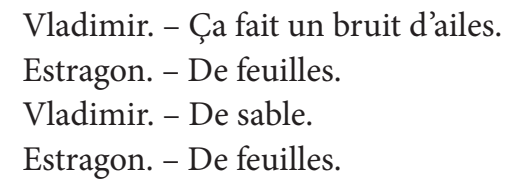

Silence.

Vladimir. - Elles parlent toutes en même temps.

Estragon. - Chacune à part soi.

Silence.

Vladimir. - Plutôt elles chuchotent.

Estragon. - Elles murmurent.

Vladimir. - Elles bruissent.

Estragon. - Elles murmurent.

Silence. (Beckett, $1952: 88)$.

\subsection{Autoriser l'espoir}

Si le silence peut, comme l'évoque Alain Corbin, renvoyer au "silence de Dieu " et donc à son absence, générant par là une angoisse profonde, on peut aussi faire l'hypothèse que chez Beckett, il ouvre l'espace des possibles :

Dans le passé, les hommes d'Occident goûtaient la profondeur et les saveurs du silence.

Ils le considéraient comme la condition du recueillement, de l'écoute de soi, de la création ; surtout comme le lieu intérieur d’où la parole émerge (Corbin, 2016 : 9).

Le silence peut donc se charger d'une fonction salvatrice aussi dans son rapport à l'espoir. Les pauses induisent une attente qui, tant qu'elle n'a pas été déçue, contient en creux tous les fantasmes de joie et de salut. Le silence permet tout d'abord, même si cela semble contradictoire, de relancer le dialogue. L'un est toujours en attente de l'autre. L'espoir est contenu dans ce silence pour permettre à l'autre d'y insérer sa propre parole. Il crée ainsi un espace de désir, une main tendue vers le vide pour échapper à la solitude, ce que souligne cette réplique d'Estragon :

Ce serait là en effet un grave inconvénient. (Un temps.) N'est-ce pas, Didi, que ce serait là un grave inconvénient ? (Un temps.) Étant donné la beauté du chemin. (Un temps.) Et la bonté des voyageurs. (Un temps. Câlin.) N'est-ce pas, Didi ? (Beckett, 1952 : 20).

Le personnage attend qu'on lui réponde, cherche la relance et, par conséquent, espère. Tout devient possible, puisque rien n’a encore été dit.

Le silence devient aussi le marqueur d'une solidarité, d'une compassion collective, puisqu'il annonce quelque chose à venir. Même si cet à-venir n'est réduit qu'à une éventualité, le silence porte cet espoir qui dure tant que le dialogue est suspendu, tant que l'interlocuteur n'a pas encore répondu, ce qui se manifeste dans la multiplication de l'interjection « hein » que l'on retrouve notamment dans Oh les beaux jours 
et Godot: «On n’est pas lié? (Un temps.) Hein ?». On perçoit ici tout le désir de la parole de l'autre qui viendrait apaiser la souffrance d'une parole solitaire.

Enfin, l'attente du divin peut s'incarner dans ces pauses répétées, davantage dans Godot, où les personnages sont confrontés à l'absence de ce Monsieur Godot qui ne se présente jamais. Le silence peut renvoyer ici à l'espoir en germe d'une manifestation divine. Rien ne se passe mais ce rien n'est pas le néant. Il est déjà quelque chose, puisqu'il est rien. On peut donc émettre l'hypothèse que tout est à construire dans cet espace vide, que l'imaginaire des deux larrons peut remplir cette béance pour se consoler d'une présence absente. Le silence matérialise alors l'inexprimable, mais aussi l'imaginable. Tout peut arriver. Et en cela, au contraire du surgissement, on peut parler d'engloutissement du néant : " [1]'inexprimable ne se veut donc pas renoncement, mais obstacle surmonté puisque assumé » écrit Julia Siboni (2010 : 18).

Pour Beckett, la parole abîme le silence. À l'effondrement du verbe, à la mutilation du langage, il faut donc substituer l'ostentation du geste, il faut faire parler le corps. $\mathrm{Si}$ le silence incarne l'angoisse existentielle des personnages confrontés à leur finitude, il peut toutefois avoir une véritable fonction salutaire : il permet d'abord de mettre en lumière l'inefficacité du langage, d'en faire surgir toute sa vacuité. D'autre part, comme l'exprime Pascale Alexandre-Bergues, dans cette alternance entre aphasie et logorrhée, "le silence concurrence sans doute la parole mais il est aussi ce qui, parfois, lui donne sa pleine résonance, isolant des mots dont il fait miroiter la beauté insolite» (2011 : 996). Enfin, le silence ouvre l'espace du possible, des possibles, justement, parce qu'il n'est que suspension, donc attente.

\section{BIBLIOGRAPHIE}

Alexandre-Bergues P. 2011. Silence. In Hubert M.-C. Dictionnaire Beckett. Paris. Honoré Champion Éditeur. 992-996.

Artaud A. 1964. Le théâtre et son double. Paris. Éditions Gallimard. coll. « Idées ».

Beckett S. 1952. En attendant Godot. Paris. Éditions de Minuit.

Beckett S. 1957. Fin de partie. Paris. Éditions de Minuit.

Beckett S. 1959. La dernière bande. Paris. Éditions de Minuit.

Beckett S. 1963. Oh les beaux jours. Paris. Éditions de Minuit.

Beckett S. 1978. Esquisse radiophonique. Paris. Éditions de Minuit.

Beckett S. 1995. Eleutheria. Paris. Éditions de Minuit.

Corbin A. 2016. Histoire du silence. Paris. Éditions Albin Michel.

Ionesco I. 1967. Journal en miettes. Paris. coll. « Folio essais».

Jacquart E. 1998. Le théâtre de dérision. Paris. Éditions Gallimard.

Parisse L. 2008. La "parole trouée ». Caen. Lettres Modernes Minard.

Siboni J. 2010. Inhumanité trop humaine ou comment Beckett persiste à nommer l'innommable. In Brown L. (éd.). Samuel Beckett 2: Parole, regard et corps. Caen. Lettres Modernes Minard. 13-26.

Vigier L. 2010. (Un temps.). In Dubor F. et Guilbard A.-C. Beckett, le mot en espace. Rennes. Presses universitaires de Rennes. 63-70. 


\section{Silence aches in Beckett's theatre}

ABSTRACT: The silence in Beckett's plays can be interpreted in many different ways. It often shows the anxiety of the characters faced with the vacuity in their lives. Left to themselves, they hardly manage to let go during these recurring silences (marked in an obsessional way in Beckett's texts with the word "pause" as an absolute punctuation in the theatrical language). So they really feel the silence as the "arising of nothingness", a sort of gateway to finitude.

This silence is also the one appearing among Beckettian couples to reveal the aporia in language: inability to communicate, "doing" instead of (impossible) "saying". This Beckettian "doing" is shown in a conspicuous gestuality which conveys a certain materiality to this silence as well as it tries desperately to fill it. Thus Beckett's characters act and give silence some substance, incarnating therefore a full-fledged character.

Finally, silence can also embody the religious, at least the expectation (of the divine? in Godot particularly?). This silence grows solemn and reveals a suspension in the speech and characters in search of a follow-up. Silence then becomes the opening of an area where everything is possible since nothing has been said yet, implicitly expressing fantasies of joy and salvation.

Keywords: silence, aporia, nothingness, gestuality, salvation. 